\title{
RELATO DE \\ Projeto-piloto do Primeiro Inquérito Nacional de Populações Expostas a Substâncias Químicas, 2008-2009*
}

doi: $10.5123 /$ S1679-49742014000300019

\section{Pilot study of the First National Survey of Populations Exposed to Chemicals, 2008-2009}

Nelson Gouveia

Departamento de Medicina Preventiva, Universidade de São Paulo, São Paulo-SP, Brasil

Rúbia Kuno

Divisão de Toxicologia Humana e Saúde Ambiental, Companhia Ambiental do Estado de São Paulo, São Paulo-SP, Brasil

Carmen Fróes Asmus

Instituto de Estudos de Saúde Coletiva, Universidade Federal do Rio de Janeiro, Rio de Janeiro-RJ, Brasil

Paulo Rubens Guimarães Barrocas

Escola Nacional de Saúde Pública Sérgio Arouca, Fundação Oswaldo Cruz, Rio de Janeiro-RJ, Brasil

Vera Regina Rossi Lemes

Instituto Adolfo Lutz, Coordenadoria de Controle de Doenças, Secretaria de Estado da Saúde, São Paulo-SP, Brasil

Anamaria Testa Tambellini

Instituto de Estudos de Saúde Coletiva, Universidade Federal do Rio de Janeiro, Rio de Janeiro-RJ, Brasil

Armando Meyer

Instituto de Estudos de Saúde Coletiva, Universidade Federal do Rio de Janeiro, Rio de Janeiro-RJ, Brasil

Volney de Magalhães Câmara

Instituto de Estudos de Saúde Coletiva, Universidade Federal do Rio de Janeiro, Rio de Janeiro-RJ, Brasil

Josino Costa Moreira

Escola Nacional de Saúde Pública Sérgio Arouca, Fundação Oswaldo Cruz, Rio de Janeiro-RJ, Brasil

Andréa Gomes de Oliveira

Departamento de Formação Específica em Fonoaudiologia, Universidade Federal Fluminense, Nova Friburgo-RJ, Brasil

Juliana de Rezende Chrisman

Escola Nacional de Saúde Pública Sérgio Arouca, Fundação Oswaldo Cruz, Rio de Janeiro-RJ, Brasil

Valesca Alves Cavalcanti

Escola Nacional de Saúde Pública Sérgio Arouca, Fundação Oswaldo Cruz, Rio de Janeiro-RJ, Brasil

Tereza Atsuko Kussumi

Instituto Adolfo Lutz, Coordenadoria de Controle de Doenças, Secretaria de Estado da Saúde, São Paulo-SP, Brasil

Viviane Emi Nakano

Instituto Adolfo Lutz, Coordenadoria de Controle de Doenças, Secretaria de Estado da Saúde, São Paulo-SP, Brasil

Sonia Bio Rocha

Instituto Adolfo Lutz, Coordenadoria de Controle de Doenças, Secretaria de Estado da Saúde, São Paulo-SP, Brasil

Maria Celeste Cardeal de Oliveira

Instituto Adolfo Lutz, Coordenadoria de Controle de Doenças, Secretaria de Estado da Saúde, São Paulo-SP, Brasil

Iracema de Albuquerque Kimura

Instituto Adolfo Lutz, Coordenadoria de Controle de Doenças, Secretaria de Estado da Saúde, São Paulo-SP, Brasil

Fernando Barbosa

Laboratório de Toxicologia e Essencialidade de Metais, Faculdade de Ciências Farmacêuticas de Ribeirão Preto, Universidade de São Paulo, Ribeirão Preto-SP, Brasil

* Projeto financiado pelo Ministério da Saúde - Convênio n 2507/2007.

Endereço para correspondência:

Nelson Gouveia - Departamento de Medicina Preventiva, Faculdade de Medicina, Universidade de São Paulo, Av. Dr. Arnaldo, $\mathrm{n}^{\circ}$ 455, São Paulo-SP, Brasil. CEP: 01246-903.

E-mail:ngouveia@usp.br 


\title{
Resumo
}

Para viabilizar a futura realização de um Inquérito Nacional de Populações Expostas a Substâncias Químicas, executou-se um projeto-piloto para examinar a exequibilidade, testar e adequar metodologias e estabelecer parcerias. Foram realizados três estudos de corte transversal, respectivamente, com doadores de sangue residentes na Região Metropolitana de São Paulo-SP, conscritos do Exército Brasileiro e crianças escolares residentes no município do Rio de Janeiro-RJ, mediante aplicação de questionários e coleta de material biológico (sangue venoso e capilar, unha e cabelo) para análise de metais e compostos organoclorados persistentes. Concluiu-se que é viável a realização de um Inquérito Nacional, embora a estratégia de obtenção das amostras biológicas deva ser definida para cada subgrupo da população. Recomenda-se aproveitar as estruturas de serviços existentes para obtenção de amostras, utilizar matrizes tradicionais para maior comparabilidade e garantir sua realização periódica

Palavras-chave: Poluentes Químicos; Monitoramento Epidemiológico; Exposição Ambiental; Saúde Pública.

\begin{abstract}
To enable the future establishment of a National Survey of Populations Exposed to Chemical Substances, a pilot study was conducted to assess the feasibility, to test and adjust methodologies, and to establish partnerships. Three crosssectional studies were performed: with blood donors residents in the Metropolitan Region of São Paulo, Brazilian army conscripts and schoolchildren living in the city of Rio de Janeiro, by applying a questionnaire and collecting biological material (capillary and venous blood, nail and hair) for the analysis of metals and persistent organochlorine compounds. We concluded that it is feasible to carry out a National Survey, but the strategy for obtaining the biological samples must be set for each sub-group of the population. Moreover, it is recommended to take advantage of the structures of existing services for obtaining samples to maintain the comparability and ensure their periodicity.
\end{abstract}

Key words: Chemical Pollutants; Epidemiological Monitoring; Environmental Exposure; Public Health.

\section{Introdução}

A saúde humana desenvolve-se e mantém-se graças à interação entre o homem e o meio ambiente. Para que a saúde seja protegida, o ambiente deve estar ajustado de maneira adequada. A partir do século passado, com a expansão da indústria e da agricultura, o homem viu-se exposto a um número cada vez maior de substâncias químicas, tanto no ambiente de trabalho como em seu macroambiente. Em vista disso, torna-se importante realizar estudos sobre contaminantes químicos ambientais, haja vista a severidade dos efeitos tóxicos e riscos que oferecem para a saúde humana.

A mensuração periódica de determinada substância química ou seu metabólito na população, conhecida como biomonitorização humana (BH), tem sido um importante instrumento para avaliar a exposição humana a substâncias químicas presentes em ambiente externo ou de trabalho, além de indicar o quanto das substâncias químicas foi realmente absorvido do ambiente pelo homem. Desse modo, a BH constituise na forma mais direta para identificar e quantificar exposição e risco, entender os mecanismos dos efeitos tóxicos e decidir sobre as medidas a serem adotadas para reduzir essa exposição. Os países que utilizam a
BH não somente monitoram a exposição da população geral aos poluentes ambientais como também avaliam o estado de saúde da população. Nos últimos anos, esses estudos têm crescido de tal forma que vêm sendo utilizados para estabelecer e revisar limites de exposição ocupacional, e para fornecer dados sobre exposição nos processos de avaliação de risco. ${ }^{1}$

\section{A mensuração periódica de determinada substância química ou seu metabólito na população, conhecida como biomonitorização humana (BH), tem sido um importante instrumento para avaliar a exposição humana a substâncias químicas.}

Acompanhando a tendência mundial, também no Brasil, a BH está se configurando como instrumento para a tomada de decisões na área da Saúde no Brasil. Em 2006, iniciaram-se discussões - conduzidas pela Secretaria de Vigilância em Saúde do Ministério da Saúde, por meio de sua Coordenação-Geral de Vigilância em Saúde Ambiental (CGVAM/SVS/MS) para 0 estabelecimento de um Inquérito Nacional de Populações Expostas a Substâncias Químicas, com 
os objetivos de (i) avaliar o nível da exposição dos brasileiros a substâncias químicas de interesse para a Saúde Pública e (ii) identificar grupos da população sob maior risco, devido à exposição aumentada. Desse modo, poder-se-ão subsidiar ações de vigilância cada vez mais efetivas, tendo em vista a possibilidade de identificação de prioridades quanto às substâncias avaliadas e as características das populações submetidas a tais exposições.

Instituições acadêmicas e de pesquisa, articuladas com a CGVAM/SVS/MS, elaboraram um estudo-piloto para testar e adequar metodologias, estabelecer parcerias, identificar limitações e principais obstáculos, de maneira a avaliar a exequibilidade da realização do Primeiro Inquérito Nacional de Populações Expostas a Substâncias Químicas. 0 presente artigo relata a experiência realizada e discute alguns dos principais problemas encontrados na perspectiva de subsidiar a implementação de um primeiro inquérito.

\section{O estudo-piloto}

0 estudo constituiu-se de três subprojetos, focados em populações específicas: doadores de sangue residentes na Região Metropolitana de São Paulo-SP; conscritos do Exército Brasileiro residentes no município do Rio de Janeiro-RJ; e crianças matriculadas em escolas da rede municipal de ensino do mesmo Rio de Janeiro-RJ. Entre os doadores e conscritos do Exército, foram coletadas amostras de sangue total e cabelo. Entre as crianças, foram coletadas amostras de sangue capilar e de unha. Nas amostras biológicas, foram realizadas análises de metais-traço (chumbo, cádmio, mercúrio, níquel etc.) e resíduos de agrotóxicos organoclorados: $\mathrm{HCB}$, alfa $\mathrm{HCH}$, beta HCH, gama HCH, op' e pp'DDT, op' e pp'DDE, op' e pp'DDD, heptacloro, heptacloro epóxido, dieldrin, dodecacloro (neste caso, apenas sobre as amostras de soro dos conscritos e doadores de sangue). Por fim, um questionário foi aplicado a cada participante (e aos pais ou responsáveis pelos escolares), para coleta de informações sociodemográficas, hábitos pessoais e possíveis exposições ambientais e ocupacionais.

\section{Amostras selecionadas}

Um inquérito nacional deve considerar uma amostra representativa da população, incluindo crianças, jovens, adultos e idosos. Este projeto-piloto buscou explorar a exequibilidade e a representatividade de três populações diferentes e, ao mesmo tempo, examinar as dificuldades e facilidades que cada uma delas apresenta.

A amostra de doadores de sangue mostrou ser uma alternativa atraente do ponto de vista logístico, pela facilidade de obtenção das amostras biológicas. Além disso, os doadores de sangue amostrados apresentaram perfil bastante semelhante ao da população adulta de referência, seja em relação à raça, local de nascimento ou variáveis socioeconômicas. Notou-se, porém, um ligeiro predomínio da população masculina $(64,7 \%)$.

A inclusão de conscritos do Exército mostra-se conveniente por esses jovens se encontrarem em uma faixa etária intermediária, de difícil acesso pelos inquéritos tradicionais. Embora não neste projeto-piloto, no qual essa dificuldade com a faixa etária é superada: todos os jovens nessa idade são obrigados a comparecer às comissões de seleção para o serviço militar. Como se trata apenas de jovens do sexo masculino de uma faixa etária bastante restrita ( $97 \%$ dos sujeitos da pesquisa tinham de 18 a 20 anos), sua representatividade é limitada.

Problema igualmente considerável se aplica às crianças. Mais acessíveis em suas escolas, elas podem representar bastante bem essa faixa etária; porém, sua participação em inquéritos dessa natureza esbarra em questões logísticas importantes a serem consideradas. Por exemplo, é necessária uma boa articulação com os gestores municipais da área da Educação, diretores e professores, além da disponibilidade da própria escola para receber as equipes de pesquisa. A marcação do período de coleta dos dados deve obedecer às especificidades próprias da organização do sistema escolar: carga horária diária de aulas, recessos, férias e períodos de provas.

A realização de um estudo abordando crianças também exige a obtenção do consentimento dos responsáveis por elas. A rotina de trabalho da maioria dos pais/responsáveis, entretanto, dificulta sua participação na vida escolar dos filhos, como por exemplo, o comparecimento às reuniões marcadas para esclarecimento e assinatura do Termo de Consentimento Livre e Esclarecido para participação das crianças no estudo. A existência de uma associação de pais em uma das escolas amostradas revelou-se decisiva para a maior divulgação e melhor esclarecimento quanto aos objetivos do estudo, e consequente aumento de dados coletados. 
Quaisquer que sejam os grupos populacionais priorizados em um inquérito nacional, é preciso estabelecer estratégias claras para a obtenção de amostras. Além disso, a exemplo de outros países, é importante que o grupo populacional definido seja o mesmo ao longo dos anos, no sentido de garantir comparabilidade entre os dados, coletados em diferentes momentos.

\section{Matriz biológica: adequação,}

\section{coleta, armazenamento e transporte}

Para a realização de um inquérito nacional, devese decidir o marcador biológico de exposição mais adequado. 0 tipo de amostra deve ser considerado em relação a suas especificidades, para a avaliação de sua exposição ao contaminante de interesse, facilidade de obtenção e exequibilidade analítica. É mister lembrar que um biomarcador eficiente deve apresentar alta sensibilidade, alta especificidade, ser biologicamente relevante e factível (tanto prática como economicamente).

Foram testadas as matrizes 'sangue total', 'soro', 'sangue capilar', 'cabelo' e 'unha' entre as diferentes populações examinadas. As matrizes 'soro' e 'sangue total', historicamente mais utilizadas em inquéritos dessa natureza, permitem maior facilidade de comparação dos resultados obtidos com os de vários estudos sobre organoclorados e metais, já publicados. Apesar de sua tradição, a coleta de amostras de sangue implica maiores dificuldades logísticas e de aceitação, principalmente entre a população infantil. Por essa razão, explorou-se a coleta de sangue capilar como alternativa ao sangue venoso, amostras de unha de crianças, e amostras de cabelo de jovens conscritos e adultos.

O manuseio do material utilizado para a coleta do sangue capilar foi fácil, obtendo-se, progressivamente, maior quantidade de amostras na medida da prática adquirida. Contudo, a análise de seus resultados foi prejudicada pela insuficiência de estudos que houvessem utilizado essa matriz para análise de metais, o que prejudica a interpretação dos resultados.

A coleta das unhas apresentou dificuldades. Algumas crianças tinham unhas muito curtas, impedindo que fossem cortadas. Do mesmo modo, faltam estudos indicativos da comparabilidade dessa matriz com outras, como sangue venoso, de modo a seus resultados serem validados.
A coleta de cabelo, de maneira geral, é realizada com facilidade por não ser invasiva ou causar desconforto, sendo mais aceita pelos sujeitos das pesquisas que a coleta de sangue. Algumas situações, por sua vez, podem tornar mais complicada sua obtenção, como no caso de militares ou pessoas com elevado grau de calvície, sem quantidade suficiente de cabelo para uma amostragem adequada. Do ponto de vista de seu armazenamento e transporte, amostras de cabelo são bastante convenientes: por serem amostras sólidas, cuja integridade não é facilmente alterada, podem ser armazenadas e transportadas sem a exigência de maiores cuidados.

As análises preliminares sobre a existência de correlação entre os elementos medidos em sangue e em cabelo mostraram uma modesta correlação apenas para o mercúrio. Além disso, há dúvidas sobre o real significado da presença desses elementos no cabelo, recomendando-se parcimônia na utilização dessa matriz para pesquisa de contaminantes. A análise de cabelo não é um método válido para determinar a exposição individual a contaminantes ambientais, exceto para metilmercúrio, ${ }^{2}$ opinião compartilhada por outros pesquisadores. ${ }^{3}$ Em muitos casos, os resultados das análises de cabelo não podem ser usados para determinar a fonte de exposição ao agente tóxico nos grupos estudados, devido ao fato de concentrações elevadas no cabelo poderem resultar de incorporação interna, via transferência da matriz sangue para 0 cabelo, como também por contaminação externa, via ar, cosméticos ou água.

Assim, sob o aspecto da logística de coleta, armazenamento e envio para os laboratórios específicos, deve-se estabelecer um plano muito bem definido e com pessoal comprovadamente capacitado, tanto para obtenção da amostra como para seu armazenamento e transporte. Uma possibilidade de garantia da segurança nesses procedimentos consiste no envolvimento, para sua execução, de pessoal dos próprios quadros dos serviços de saúde com experiência em coleta e armazenamento de material biológico.

As condições adequadas de armazenamento variaram de acordo com o analito estudado e a matriz coletada. De todo modo, sempre buscou-se evitar condições que propiciassem alterações físicas ou químicas das amostras, como evaporação, precipitação, hemólise, degradação do analito de interesse etc. Quase todas as amostras foram armazenadas sob 
refrigeração $\left(<5^{\circ} \mathrm{C}\right)$, em frascos identificados e hermeticamente fechados. No caso da análise de metais, faz-se necessária a presença de agentes anticoagulantes nos recipientes de coleta, para evitar a perda dos metais-traço.

\section{Definição dos laboratórios}

Um dos maiores obstáculos à realização desse tipo de inquérito, identificado durante o estudo-piloto, é a inexistência de uma rede nacional de laboratórios com comprovada capacidade técnica para realizar as análises em quantidade e qualidade necessárias. Os laboratórios públicos, sobretudo os Laboratórios Centrais de Saúde Pública (LACEN), deverão ser priorizados no esforço de estruturação dessa rede, a qual deverá incluir tanto a adequação da infraestrutura laboratorial, eventualmente com a aquisição de equipamentos analíticos modernos, como a qualificação e capacitação de mão de obra necessária à realização dessas análises. Tornar-se-á, ainda, indispensável a organização sistemática de exercícios de intercalibração entre os laboratórios que participarem dessa rede, para a garantia e o controle da qualidade das análises. Essa rede poderá ser administrada pela Coordenação-Geral de Laboratórios de Saúde Pública (CGLAB/SVS/MS), contando - preferivelmente - com a participação de instituições internacionais. Se forem utilizados laboratórios privados, estes deverão ser submetidos a acreditação (ISO 17025)/Monitoramento BPL, e passíveis de auditoria. 0 repasse de verba para as análises do inquérito deverá ser feito anualmente, incorporado à previsão orçamentária do Ministério da Saúde (considerando-se que o inquérito será um processo contínuo).

\section{Questionário}

Além da caracterização sociodemográfica e das variáveis levantadas como ocupações passadas com uso de agrotóxicos, criação de animais, horta e fonte de água para consumo, morar próximo a lixo industrial ou em região agrícola, algumas questões importantes deverão ser incluídas no questionário a ser aplicado em um inquérito nacional. 0 tempo médio de residência no(s) último(s) endereço(s) constitui informação capaz de auxiliar na definição de possíveis exposições passadas. 0 número de restaurações de amálgama na cavidade bucal é um dos principais fatores de risco para aumento dos níveis de Hg em sangue. Outra informação a ser obtida refere-se ao consumo de pescado nas últimas 48 horas, importante fator de risco para presença de arsênio (As) no organismo. A entrevista direta com as crianças apresentou deficiência na obtenção de algumas informações básicas, por falta de conhecimento. Os questionarios foram enviados para preenchimento em casa e seu retorno foi inferior - em números - ao de amostras de sangue.

\section{Conclusões}

- é viável a realização de um Inquérito Nacional de Populações Expostas a Contaminantes Químicos, embora a estratégia de obtenção das amostras biológicas deva ser definida para cada subgrupo da população;

- pode-se aproveitar as estruturas de serviços existentes para obtenção de amostras, como bancos de sangue e o sistema público de saúde;

- pode-se utilizar matrizes tradicionalmente usadas nesses estudos, como sangue e urina, para garantir a comparabilidade, sendo possível incluir outras matrizes com o objetivo de formar banco de dados históricos;

- deve-se buscar a estruturação de uma rede de laboratórios adequada ao desafio de uma participação em inquéritos nacionais; $\mathrm{e}$

- a implantação de um inquérito nacional deve ser feita de maneira a garantir sua continuidade, pois os dados obtidos devem refletir as condições ambientais.

\section{Agradecimentos}

A Irani Cristiane da Silva e Valéria Aparecida Cunha Diago, bolsistas de pesquisa ligadas a este projetopiloto.

A Reinaldo Amauri Ribeiro e Antonia de Lima Silva, pelo apoio técnico.

E às instituições colaboradoras: Associação Beneficente de Coleta de Sangue (Colsan) de São Paulo Secretaria Municipal de Educação do Rio de Janeiro e Direção das escolas CIEP Yuri Gagarin e Escola Desembargador Ney Palmeiro e Exército Brasileiro.

\section{Contribuição dos autores}

Gouveia N, Kuno R, Asmus CF, Barrocas PRG, Lemes VRR e Aguiar AGO contribuíram com a concepção e 
delineamento do estudo, análise e interpretação dos dados, e redação do manuscrito.

Tambellini AT, Meyer A, Câmara VM e Moreira JC contribuíram com a concepção e delineamento do estudo.

Chrisman JR e Cavalcanti VA contribuíram com a coleta de dados.

\section{Referências}

1. Levy LS, Jones K, Cocker J, Assem FL, Capleton AC. Background levels of key biomarkers of chemical exposure within the UK general population - pilot study. Int J Hyg Environ Health. 2007 May;210 (34):387-91.

2. Harkins DK, Susten AS. Hair analysis: exploring the state of science. Environ Health Perspect. 2003 Apr;111(4):576-8.
Kussumi TA, Nakano VE, Rocha SOB, Oliveira MCC, Kimura IA e Barbosa F contribuíram com as análises químicas e toxicológicas.

Todos os autores aprovaram a versão final do manuscrito e declararam-se responsáveis por todos os aspectos do trabalho, garantindo sua precisão e integridade.

3. Wilheelm M, Idel H. Hair analysis in environmental medicine. Int J Hyg Environ Health. 1996 Jul;198(6):485-501.

\section{Recebido em 06/05/2014}

Aprovado em 01/08/2014 